\section{Semen polyamines in AIDS pathogenesis}

SIR - Recent correspondence has drawn attention to the immunosuppressive properties of cellular and soluble constituents of human semen which may be significant in the aetiology of the acquired immune deficiency syndrome (AIDS) ${ }^{1-3}$. The discovery of spermatozoa in semen by Antoni van Leeuwenhoek in 1677 was accompanied by a description of small crystals deposited from seminal fluid ${ }^{4}$ which are now known to be composed of spermine phosphate. Spermine is present in seminal plasma at concentrations between 5 and 15 $\mathrm{mM}$ together with lower concentrations of another polyamine, spermidine, and their biosynthetic precursor, the diamine putrescine $^{5}$. Micromolar quantities of either polyamine have been shown to inhibit the in vitro responses of murine lymphocytes assayed by mitogen stimulation, mixed lymphocyte reactions and cytolytic responses ${ }^{6}$. Such inhibitory effects were shown to be reversible but they occurred only in the presence of calf or fetal calf sera.

Polyamine oxidase activity in ruminant sera may form aminoaldehydes and acrolein which are known to be toxic to cells in culture. Inhibitors of spermine oxidase have been shown to abolish the suppression of lipopolysaccharide-mediated mitogenesis of murine lymphocytes produced by fetal calf serum and polyamines but all suppression was not necessarily due to cytotoxicity ${ }^{7}$. Indeed, the DNA synthetic response of human lymphocytes stimulated by phytohaemagglutinin can be inhibited by spermine or spermidine in the presence of fetal calf serum without apparent cytocidal effects as measured by Trypan blue exclusion (V. Tan and J.D.W., in preparation). Another recent study has shown that the fully acetylated diamines hexamethylenebisacetamide and diacetylputrescine can cause inhibition of murine B lymphocyte activation in the absence of ruminant serum ${ }^{8}$. Thus, although their mechanism of action remains unknown, the polyamines and diamine in human semen can be implicated experimentally in immunosuppression.

The postulated sequelae of the exposure to high concentrations of polyamines in human semen could be exacerbated if similar immunosuppressive mechanisms arise from infections commonly found in AIDS patients. Seroepidemiological studies have shown antibodies to human cytomegalovirus (CMV) in $94 \%$ of male homosexuals ${ }^{9}$ and 14 out of 15 autopsied AIDS cases in one study had disseminated CMV infections ${ }^{10}$. Infection of MRC 5 cells with human CMV results in increased polyamine biosynthesis leading to spermine levels 12 -fold higher than in uninfected control cells ${ }^{11}$. These virusinduced metabolic changes can be calculated to produce enhanced accumulation of polyamines sufficient to be in- hibitory in terms of the lymphocyte function assays described above. However, other in vivo immunomodulatory effects mediated by polyamines can be envisaged. It is known that exogenous putrescine can restore 'suppressor' cell activity associated with the regulation of haematopoiesis after polyamine depletion in mice treated with the specific inhibitor DL- $\alpha$-difluoromethylornithine $^{12}$. A similar requirement for polyamines in the modulation of accessory cell activity in the immune system may influence $\mathrm{T}$-suppressor cell activity in the presence of exogenous polyamines. Immunosuppression is a significant feature of human CMV infection and activation of $T$-suppressor cells has been shown in cytomegalovirus mononucleosis ${ }^{13}$.

Lymphotropic retroviruses have recently been isolated specifically from AIDS patients but it is still not clear if such infections alone can account fully for the immunopathogenesis of the disease ${ }^{14}$. If other co-factors are required, various mechanisms of immunosuppression can be proposed which are mediated by components of semen found in the high-risk group. Since human CMV is present in semen, in addition to saliva and urine, such body fluids constitute a potent source of infection. The presence of polyamines in semen and their further production during human CMV infection may be significant aetiological factors in the pathogenesis of AIDS.

Division of Virology,

Department of Medical Microbiology, St Mary's Hospital Medical School, London W2 IPG, UK

1. Shearer, G.M. \& Rabson, A.S. Nature 308, 230 (1984)

. Olsen, G.P. \& Shields, J.W. Nature 309, 116 (1984)

James, K. Nature 309, 117 (1984).

. van Leeuwenhoek, A. Phil. Trans. R. Soc. 12, 1040 (1678).

Williams-Ashman, H.G. \& Lockwood, M.D. Ann. N.Y. Acad. Sci. 171, 882 (1970).

6. Byrd, W.J., Jacobs, D.M. \& Amoss, M.S. Nature 267, 621 (1977)

7. Labib, R.S. \& Tomasi, T.B. Eur. J. Immun. 11, 266 (1981)

8. Ryan, J.I., Bondy, P.K., Gobran, L. \& Canellakis, Z.K. J Immun. 132, 1888 (1984)

9. Drew, W.L. et al. Lancet ii, 125 (1982)

0. Macher, A.M. et al. New. Engl. J. Med. 309, 1454 (1983) Tyms, A.S. \& Williamson, J.D. Nature 297, 690 (1982).

Sharkis, S.J. et al. Blood 61, 604 (1983).

13. Carney, W.P. et al. J. Immun. 126, 2114 (1981).

14. Lancet i, 1053 (1984).

SIR - I would like to acknowledge an oversight in our recent letter (Nature 308, 230; 1984), in which we did not, but should have cited Mavligit et al. (J. Am. med. Ass. 251, $237 ; 1984)$ for pointing out the difference between the epithelial lining of the vagina and the rectum.

GENE M. SHEARER

National Institutes of Health,

A. RABSON

National Cancer Institute,

Bethesda, Maryland 20205, USA

\section{Caution on AIDS viruses}

SIR - Since human T-cell leukaemia virus (HTLV) 1 and HTLV 2 have been isolated from patients with acquired immune deficiency syndrome (AIDS), there are now at least three or possibly four T lymphotropic viruses that can be associated with this syndrome (if lymphadenopathy-associated virus proves to be different from HTLV 3 ).

If at least three $\mathrm{T}$-cell tropic viruses are recoverable from AIDS patients, albeit with different frequencies, it suggests that they are more likely to represent opportunistic infections or reactivations from latency. This is not to say that they play no role in the overall pathogenesis of AIDS.

The problem of so many isolates is not mitigated by a possible relationship between them, nor by differences in isolation frequency. This difficulty does not seem to bother Dr Groopman (Nature 308, 769; 1984), who appears satisfied that the causation of AIDS has been revealed.

AIDS is indeed a tragic disease, as Dr Groopman notes. This tragedy can only be compounded by premature interpretations of the role of new viral isolates with the promise of a vaccine, and a diminished focus on other possible aetiologic factors.

49 West 12th Street

J.A. SONNABEND

New York,

New York 10011, USA

\section{Somatic gene mutation and breast carcinoma}

SIR - In her crisp summary on the new information concerning recessive mutations in Wilm's tumour and retinoblastoma and her presentation of likely mechanisms of their evolution, Ellen Solomon (Nature 309,$111 ; 1984$ ) suggests the possibility that similar mechanisms may lie behind the more common carcinomas of late onset and these should now be explored. She points out that any such efforts are hampered by the "haystack" problem because of our lack of knowledge about which chromosome to study with DNA probes. The challenge of finding out if the tumour cells contain increased hemi- or homozygosity is reasonable when genes are pinpointed by the breakpoint of a specific chromosome change but, as Ellen Solomon stresses, it is often difficult to obtain adequate preparations from carcinomas so it is not yet known if they carry specific chromosome abnormalities.

We have recently tried to overcome these problems and fill the gap in our knowledge of breast carcinoma by cytogenetic investigations using consecutive banding techniques on direct chromosome preparations from 111 breast carcinomas obtained at mastectomy from 110 women and 1 man (Rodgers et al. Cancer Genet. Cytogenet., in the press).

All tumour samples which contained cells in mitosis $(52 / 111,47 \%)$ showed chromosome abnormalities which in a high 